\title{
Elargissement du réseau de nos sociétés fiduciaires partenaires dans les cantons de Neuchâtel et Fribourg
}

FMH Services présente un nouveau partenaire de confiance en matière fiduciaire dans les cantons de Neuchâtel et Fribourg.

Titulaire d'un brevet fédéral de spécialiste en finance et comptabilité et réviseur agréé ASR $\mathrm{N}^{\circ} 110423$, Maxime Bugnon de la société Figemax SA est à votre disposition.

Figemax SA, société fiduciaire également agréée ASR en qualité de réviseur $\mathrm{N}^{\circ}$ 505090, vous propose des services de qualité dans les domaines suivants:

- Comptabilité

- Fiscalité personnes physiques et morales
- Business plan, études de financements

- Conseils financiers et organisationnels

- Assistance lors de la remise de cabinet

- Ressources humaines et gestion des salaires

$\mathrm{Au}$ bénéfice d'une grande expérience dans le domaine médical notamment dans la tenue comptable et fiscale auprès des professionnels de la santé, Maxime Bugnon a pour objectif de mettre ses compétences à votre service en privilégiant le dialogue.

Nous nous tenons à votre entière disposition pour tout complément d'information et nous nous réjouissons d'avance de votre prochain contact.

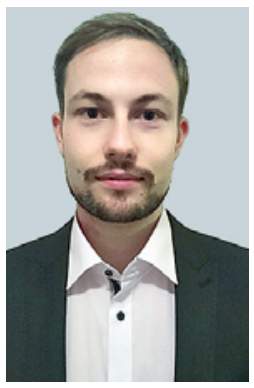

Canton de Neuchâtel

Figemax SA

FMH Services (Fiduciaire)

Quai Robert-Comtesse 3

Centre de la Maladière

2000 Neuchâtel

Tél. 0265358236
Canton de Fribourg

Figemax SA

FMH Services (Fiduciaire)

Route de la Gare 2

1680 Romont

Tél. 0265358236

Figemax SA est une entreprise de conseil indépendante sur le plan juridique et économique recommandée par la société coopérative FMH Services.

Maxime Bugnon

Spécialiste en finance et comptabilité avec brevet fédéral

maxime.bugnon@fmhfiduciaire.ch

\section{Problèmes de TVA?}

\section{ந FMHSERVICES}

«Les prestations médicales ne sont pas soumises à la TVA!» Si cette affirmation est correcte, elle ne l'est cependant pas dans tous les cas. C'est pourquoi il convient d'étudier la question en détail dans le cadre de cabinets doubles ou de groupe ou encore de la propharmacie. En tant que spécialistes, les partenaires de confiance FMH Services (Fiduciaire) se tiennent à votre entière disposition. N'hésitez pas à faire appel à eux.

\author{
FIDUCIAIRE
}

\section{FMH Consulting Services AG}

Tél. 0419250077 - Fax 0419250067

mail@fmhfiduciaire.ch - www.fmhfiduciaire.ch 